PITHA 03/08

\title{
Semileptonic decays of polarised top quarks: $V+A$ admixture and QCD corrections
}

\author{
Werner Bernreuther ${ }^{1}$, Michael Fücker ${ }^{2}$ and Yoshiaki Umeda ${ }^{3}$ \\ Institut f. Theoretische Physik, RWTH Aachen, 52056 Aachen, Germany
}

\begin{abstract}
:
The semileptonic decays of polarised top quarks are analysed for a general chiralityconserving $t b W$ vertex. We calculate double differential distributions for the charged lepton and the neutrino to order $\alpha_{s}$ in the QCD coupling. We present these QCD corrections in terms of compact parameterisations that should be useful for the future investigation of the structure of the top decay vertex on the basis of large data samples.
\end{abstract}

PACS number(s): 12.60.Cn, 13.88.+e, 14.65.Ha

Keywords: top quark decay, spin polarisation, anomalous couplings, QCD corrections

\footnotetext{
${ }^{1}$ E-mail address: breuther@physik.rwth-aachen.de

${ }^{2}$ supported by D.F.G. SFB/TR9

${ }^{3}$ supported by BMBF contract 05 HT1PAA/4
} 
So far the experimental information about the decays of top quarks is not very detailed. Data from the Tevatron are consistent with the expectation that these decays are governed by the $V-A$ charged current interactions of the standard model (SM); but because of the size of the present experimental errors sizable new physics effects can not be excluded (c.f. for instance [1]).

In view of the extremely large mass of the top quarks and the circumstance that they do not hadronise these particles are excellent probes of new interactions that may uncover at energies of a few hundred $\mathrm{GeV}$. There is a vast literature on the phenomenology of such interactions in top quark decays (for overviews, see for instance [2, 3]). One possibility is a small $V+A$ admixture to the standard lefthanded $t \rightarrow b$ current as is predicted for instance by $S U(2)_{L} \times S U(2)_{R} \times U(1)$ extensions of the standard model. The measured branching ratio of the radiative weak decay $b \rightarrow s \gamma$ provides a stringent constraint [4, 5, 6, 7, on the coupling strength $\kappa_{R}$ of such a right-handed admixture: $\left|\kappa_{R}\right| \lesssim 0.04$. Of course, one may envisage contrived scenarios where other contributions to $b \rightarrow s \gamma$ lead to cancellations that invalidate this bound - in any case, a direct search for such a coupling in $t$ and $\bar{t}$ decays is indispensable. Sensitive tests require polarised top quarks. At the LHC the single top production processes will yield large samples of polarised $t$ and $\bar{t}$ quarks, and it has been estimated [8, 9] that a statistical sensitivity $\delta \kappa_{R} \simeq 0.06$ can be reached. From $t \bar{t}$ pair production at a high-luminosity linear $e^{+} e^{-}$collider a sensitivity $\delta \kappa_{R} \simeq 0.03$ will be feasible [10, 11, 3].

Energy and angular distributions for polarised top quark decays with a $V+A$ admixture in the $t b W$ vertex were investigated in a number of articles, including [12, 13, 14, 15]. In ref. [13] semileptonic $t$ decays were analysed and it was pointed out that the polarisation-dependent part of the neutrino distribution (which can be determined experimentally by measuring the missing momentum) is very sensitive to $\kappa_{R}$. The order $\alpha_{s}$ QCD corrections for the $V-A$ part of the charged current [16, 17] were incorporated in [13]. In view of the above-mentioned expected sensitivity to $\kappa_{R}$ at future colliders one should note that taking into account QCD corrections in future data analysis is mandatory, because they can mimic a small $V+A$ admixture.

In this paper we extend the work of [13] in that we compute the order $\alpha_{s}$ QCD corrections to both the $V-A$ and $V+A$ Born contributions to $t \rightarrow b \ell \nu_{\ell}$. Moreover we take the finite width of the intermediate $W$ boson and the non-zero mass of the $b$ quark into account. The latter is important in the case of a small $V+A$ admixture, because terms linear in $\kappa_{R}$ in the lepton distributions, which we compute below, require a chirality flip of the $b$ quark. For small $\kappa_{R}$, say $\left|\kappa_{R}\right|<0.1$, the terms proportional to $\kappa_{R} m_{b} / m_{t}$ are of the same order of magnitude as the contributions proportional to $\kappa_{R}^{2}$. Furthermore we compute a T-odd triple correlation which is generated by a non-standard CP-violating phase that may be present in the general chirality-conserving $t b W$ vertex.

We consider the semileptonic decay of spin-polarised top quarks to order $\alpha_{s}$ in 
the QCD coupling, which amounts to studying the reactions

$$
\begin{aligned}
& t \rightarrow b\left(p_{b}\right)+\ell^{+}\left(p_{\ell}\right)+\nu_{\ell}\left(p_{\nu}\right) \\
& t \rightarrow b\left(p_{b}\right)+\ell^{+}\left(p_{\ell}\right)+\nu_{\ell}\left(p_{\nu}\right)+g\left(p_{g}\right) .
\end{aligned}
$$

All momenta refer to the rest frame of the top quark. As far as the $t b W$ vertex is concerned we use the generalised interaction

$$
\mathcal{L}_{t b}=-\frac{g}{2 \sqrt{2}} V_{t b} \bar{t} \gamma^{\mu}\left(\alpha-\beta \gamma_{5}\right) b W_{\mu}+\text { h.c. }
$$

where $\alpha, \beta$ are complex couplings. A right-handed admixture to the $V-A$ charged current interaction of the $\operatorname{SM}(\alpha=\beta=1)$ will be parameterised by choosing $\alpha=1+\kappa_{R}, \beta=1-\kappa_{R}$. In the calculations below we neglect the lepton masses but take the mass of the $b$ quark and the finite width of the intermediate $W$ boson into account.

In the calculation of the differential decay distributions for (1) we have performed the quark wave function renormalisations that remove the ultraviolet divergencies in the on-shell scheme. The infrared divergencies are canceled using a standard phase space slicing procedure. The phase space of the four-particle final state in the reaction $t \rightarrow b \ell \nu_{\ell} g$ is split into two disjoint regions where the (scaled) energy $x_{g}=$ $2 E_{g} / m_{t}$ of the gluon is smaller and larger than an arbitrary, but small separation parameter $x_{\text {min }}$, respectively: $1=\Theta\left(x_{\text {min }}-x_{g}\right)+\Theta\left(x_{g}-x_{m i n}\right)$. Integrating the respective squared matrix element over the phase space of the soft gluon $\left(x_{g} \leq x_{\text {min }}\right)$ and adding the result to the order $\alpha_{s}$ squared matrix element for the three particle final state $b \ell \nu_{\ell}$ yields the infrared finite differential decay distribution $d \Gamma_{B+V+s o f t}$ (B $=$ Born, $\mathrm{V}=$ virtual) and $d \Gamma_{\text {hard }}$, which describes radiation of "resolved" gluons $\left(x_{g}>x_{\text {min }}\right)$.

For checks of possible deviations from the $V-A$ law in the $t b W$ vertex useful observables are the double differential energy-angle distributions $d \Gamma / d x_{i} d \cos \theta_{i}(i=$ $\ell, \nu$ ) for the charged lepton and for the neutrino, where $x_{i}=2 E_{i} / m_{t}$ and $\theta_{i}$ is the angle between the three-momentum of the lepton $i$ and the unit vector $\hat{\mathbf{s}}$ that specifies the polarisation direction of the ensemble of top quarks in the $t$ rest frame. The degree of polarisation is denoted by $S=|\mathbf{s}|$. Adding the contributions from $d \Gamma_{B+V+\text { soft }}$ and from $d \Gamma_{\text {hard }}$, these distributions can be put into the following form:

$$
\begin{aligned}
& \frac{d \Gamma}{d x_{\ell} d \cos \theta_{\ell}}=\left|V_{t b}\right|^{2} \frac{g^{4} m_{t}}{8}\left[\left(F_{0}^{\ell n}+C_{F} \frac{\alpha_{s}}{\pi} F_{1}^{\ell n}\right)+\left(F_{0}^{\ell s}+C_{F} \frac{\alpha_{s}}{\pi} F_{1}^{\ell s}\right) S \cos \theta_{\ell}\right], \\
& \frac{d \Gamma}{d x_{\nu} d \cos \theta_{\nu}}=\left|V_{t b}\right|^{2} \frac{g^{4} m_{t}}{8}\left[\left(F_{0}^{\nu n}+C_{F} \frac{\alpha_{s}}{\pi} F_{1}^{\nu n}\right)+\left(F_{0}^{\nu s}+C_{F} \frac{\alpha_{s}}{\pi} F_{1}^{\nu s}\right) S \cos \theta_{\nu}\right],
\end{aligned}
$$

where $C_{F}=4 / 3$ and $0 \leq x_{\ell}, x_{\nu} \leq\left(m_{t}^{2}-m_{b}^{2}\right) / m_{t}^{2}$. The corresponding distributions for the anti-top quark are obtained by changing the sign of the term proportional 
to $\cos \theta_{i}$. From these distributions the corresponding $2 \times 2$ top decay spin density matrices can be extracted in straightforward fashion ${ }^{1}$.

The $F_{a}^{i j}(i=\ell, \nu, j=n, s$, and $a=0,1)$ which are functions of $x_{\ell}$ and $x_{\nu}$, respectively, can be decomposed according to the contributions from the vector and axial vector couplings $\alpha, \beta$ :

$$
F_{a}^{i j}=|\alpha|^{2} F_{a, \alpha}^{i j}+\operatorname{Re}\left(\alpha^{*} \beta\right) F_{a, \alpha \beta}^{i j}+|\beta|^{2} F_{a, \beta}^{i j} .
$$

The following relations hold between the functions that appear in the charged lepton distribution and those of the corresponding neutrino distribution:

$$
\begin{array}{ccc}
F_{a, \alpha}^{\ell n}=F_{a, \alpha}^{\nu n}, & F_{a, \beta}^{\ell n}=F_{a, \beta}^{\nu n}, & F_{a, \alpha \beta}^{\ell n}=-F_{a, \alpha \beta}^{\nu n}, \\
F_{a, \alpha}^{\ell s}=-F_{a, \alpha}^{\nu s}, & F_{a, \beta}^{\ell s}=-F_{a, \beta}^{\nu s}, & F_{a, \alpha \beta}^{\ell s}=F_{a, \alpha \beta}^{\nu s},
\end{array}
$$

where $a=0,1$. For the sake of presenting compact expressions we write down the lowest order functions of the charged lepton distribution in terms of one-dimensional integrals $(r=\alpha, \beta, \alpha \beta)$ :

$$
F_{0, r}^{\ell j}=\frac{1}{512 \pi^{3}} \int_{x_{b, \min }}^{x_{b, \max }} d x_{b} D_{W}\left(x_{b}\right) \tilde{F}_{0, r}^{\ell j}\left(x_{\ell}, x_{b}\right),
$$

where $D_{W}=\left(\left(1-x_{b}-\hat{m}_{W}^{2}+\hat{m}_{b}^{2}\right)^{2}+\hat{m}_{W}^{2} \hat{\Gamma}_{W}^{2}\right)^{-1}, \hat{m}_{W}=m_{W} / m_{t}, \hat{\Gamma}_{W}=\Gamma_{W} / m_{t}$, $\hat{m}_{b}=m_{b} / m_{t}, x_{b, \min }=\left(1-x_{\ell}+\hat{m}_{b}^{2} /\left(1-x_{\ell}\right)\right), x_{b, \max }=\left(1+\hat{m}_{b}^{2}\right)$, and

$$
\begin{aligned}
\tilde{F}_{0, \alpha}^{\ell n} & =-2 \hat{m}_{b}^{3}+\hat{m}_{b}^{2}\left(x_{b}-2\right)+2 \hat{m}_{b}\left(x_{b}-1\right)-x_{b}^{2}+x_{b}\left(3-2 x_{\ell}\right)-2\left(x_{\ell}-1\right)^{2}, \\
\tilde{F}_{0, \alpha \beta}^{l n} & =2\left(1+\hat{m}_{b}^{2}-x_{b}\right)\left(2-x_{b}-2 x_{\ell}\right), \\
\tilde{F}_{0, \beta}^{l n} & =2 \hat{m}_{b}^{3}+\hat{m}_{b}^{2}\left(x_{b}-2\right)-2 \hat{m}_{b}\left(x_{b}-1\right)-x_{b}^{2}+x_{b}\left(3-2 x_{\ell}\right)-2\left(x_{\ell}-1\right)^{2}, \\
\tilde{F}_{0, \alpha}^{l s} & =-\left(1+\hat{m}_{b}^{2}-x_{b}\right)\left(2 \hat{m}_{b}^{2}+x_{b}\left(x_{\ell}-2\right)+2\left(x_{\ell}-1\right)^{2}+2 \hat{m}_{b} x_{\ell}\right), \\
\tilde{F}_{0, \alpha \beta}^{l s} & =4\left(1+\hat{m}_{b}^{2}-x_{b}\right)^{2}+2\left(1-\hat{m}_{b}^{2}-x_{b}\right)\left(x_{b}-4\right) x_{\ell}-4\left(x_{b}-2\right) x_{\ell}^{2}-4 x_{\ell}^{3}, \\
\tilde{F}_{0, \beta}^{l s} & =-\left(1+\hat{m}_{b}^{2}-x_{b}\right)\left(2 \hat{m}_{b}^{2}+x_{b}\left(x_{\ell}-2\right)+2\left(x_{\ell}-1\right)^{2}-2 \hat{m}_{b} x_{\ell}\right) .
\end{aligned}
$$

The corresponding functions for the neutrino distribution are obtained with the relations (6). Using $\alpha, \beta=1 \pm \kappa_{R}$ it is easy to see that those terms in the Born distributions which are linear in $\kappa_{R}$ are accompanied by a factor $\hat{m}_{b}$ (or $\hat{m}_{b}^{3}$ ).

The functions $F_{1, \alpha}^{i j}, F_{1, \alpha \beta}^{i j}$, and $F_{1, \beta}^{i j}$ which are induced by the order $\alpha_{s}$ corrections are shown in Figs. 1 and 2. We use $m_{t}=174.3 \mathrm{GeV}, m_{b}=4.8 \mathrm{GeV}, m_{W}=80.42$ $\mathrm{GeV}$, and $\Gamma_{W}=2.12 \mathrm{GeV}$. For SM couplings $\alpha=\beta=1$ and for the narrow width approximation $\hat{\Gamma}_{W} \rightarrow 0$ we can numerically compare our results with those of [17] and we find agreement.

The size of the QCD corrections is typically 6 to 8 percent for $x_{i}<0.9$ and somewhat larger at the upper end of the spectrum. For $x_{i} \rightarrow 1$ the curves show the

\footnotetext{
${ }^{1}$ These density matrices enter the calculations of the (differential) cross sections that describe $t$ and/or $\bar{t}$ production and decay in the on-shell approximation.
} 


\begin{tabular}{|c||c|c|c|c|c|c|}
\hline & $F_{1, \alpha}^{\ell n}$ & \multicolumn{1}{|c|}{$F_{1, \beta}^{\ell n}$} & \multicolumn{1}{c|}{$F_{1, \alpha \beta}^{\ell n}$} & \multicolumn{1}{c|}{$F_{1, \alpha}^{\ell s}$} & $F_{1, \beta}^{\ell s}$ & $F_{1, \alpha \beta}^{\ell s}$ \\
\hline \hline$A_{0}\left[10^{-7}\right]$ & -8.06 & -10.54 & -14.37 & -7.55 & -9.86 & -15.25 \\
\hline$A_{1}\left[10^{-5}\right]$ & 5.50 & 5.50 & 4.96 & 5.24 & 5.24 & 5.18 \\
\hline$B_{0}$ & -0.2853 & -0.3087 & -1.6414 & -1.1058 & -1.2267 & -1.6760 \\
\hline$B_{1}$ & 3.3781 & 3.6545 & 22.6920 & 17.7909 & 19.7949 & 23.4286 \\
\hline$B_{2}$ & -16.3481 & -17.902 & -131.1934 & -122.4657 & -136.8286 & -136.6096 \\
\hline$B_{3}$ & 40.0362 & 44.3232 & 408.7910 & 467.4425 & 523.4506 & 428.1320 \\
\hline$B_{4}$ & -53.1628 & -59.4165 & -741.6436 & -1083.4018 & -1213.9973 & -781.1829 \\
\hline$B_{5}$ & 36.4636 & 41.0886 & 784.5948 & 1563.7047 & 1751.0343 & 831.2825 \\
\hline$B_{6}$ & -10.1118 & -11.4767 & -448.9931 & -1374.8811 & -1536.9986 & -478.5832 \\
\hline$B_{7}$ & & & 107.4585 & 674.4979 & 752.1898 & 115.2665 \\
\hline$B_{8}$ & & & & -141.6121 & -157.4561 & \\
\hline
\end{tabular}

Table 1: The coefficients which determine the fits (9) to the order $\alpha_{s}$ QCD contributions to the charged lepton distribution (3). $A_{0}$ and $A_{1}$ are given in units of $10^{-7}$ and $10^{-5}$, respectively.

developoment of the well-known logarithmic singulariy [16 which can be removed by exponentiation. Rather than presenting the exact analytical formulae for the functions $F_{1, \alpha}^{i j}, F_{1, \alpha \beta}^{i j}$, and $F_{1, \beta}^{i j}$, which are quite lengthy, we give them in terms of simple parameterisations determined by fits. We required the fits to be better than $4 \%$ in the range $0 \leq x_{\ell}, x_{\nu} \leq 0.98$. This requirement can be satisfied by using a rational function for $x_{\ell}, x_{\nu} \leq 0.218$ and a polynomial of at most 8th order for $x_{\ell}, x_{\nu}>0.218$. This number is the location of the peaks in Figs. 1 and 2 which correspond to the maximum of $D_{W}$ and the shape of the functions of $F_{1}$ around this peak arise as follows. The contributions to $F_{1}$ from $d \Gamma_{V+s o f t}$ and $d \Gamma_{\text {hard }}$ have opposite signs. The virtual and soft corrections start growing steeply at a slightly lower value of $x_{i}$ than the term from "hard" gluon radiation and the former level off slightly earlier, at $x_{i} \simeq 0.218$, where the increase of $d \Gamma_{\text {hard }}$ is still rather steep. We have checked numerically that this does not depend on the choice of the (small) separation parameter $x_{\min }$.

In the fits we use the generic form

$$
F_{1}=\Theta(0.218-x) \frac{A_{0}}{(x-0.218)^{2}+A_{1}}+\Theta(x-0.218) \sum_{k=0}^{8} B_{k} x^{k},
$$

where $\Theta(x)$ denotes the step function. The coefficients $A_{k}, B_{k}$ that specify the respective functions are given in Table 1. The corresponding functions for the neutrino distribution are obtained with the help of (6).

The polarisation dependent part of the neutrino distribution, $F^{\nu s} \equiv F_{0}^{\nu s}+$ $C_{F} \frac{\alpha_{s}}{\pi} F_{1}^{\nu s}$, is the term in the above distributions that is most sensitive to a coupling $\kappa_{R}$ [13], especially for neutrino energies $x_{\nu} \lesssim 0.6$. For instance, for $\kappa_{R}=0.1$ 
the function $F^{\nu s}$ deviates from its $\mathrm{SM}$ value by about $4 \%$ for $0.25 \lesssim x_{\nu} \lesssim 0.6$, and for $x_{\nu} \lesssim 0.25$ the deviations are even larger. In order to obtain maximal sensitivity to $\kappa_{R}$ in future data analyses one may use likelihood functions that can be derived 10. from (3) and (4).

Finally we consider the possibility that $\operatorname{Im}\left(\alpha^{*} \beta\right) \neq 0$. This happens in a natural way in $S U(2)_{L} \times S U(2)_{R} \times U(1)$ extensions of the SM, where the Higgs sector is sufficiently complicated to allow for a CP-violating phase in the $L-R$ gauge boson mixing matrix already at tree level. After diagonalisation of this mass matrix this phase is transported to the charged current interaction (2). An observable to check for such a phase is the expectation value of the T-odd triple product $\mathcal{O}=\hat{\mathbf{p}}_{\ell} \cdot\left(\hat{\mathbf{p}}_{b} \times \hat{\mathbf{s}}\right)$, where $\hat{\mathbf{p}}_{b, \ell}$ are the directions of flight of the b quark and the lepton, respectively, in the top quark rest frame. (At this order in the QCD coupling the decay amplitude has no absorptive part that could generate $\langle\mathcal{O}\rangle \neq 0$.) We have to order $\alpha_{s}$ :

$$
<\mathcal{O}>=\frac{1}{\Gamma_{S L}}\left(\int \mathcal{O} d \Gamma_{B+V+s o f t}+\int \mathcal{O} d \Gamma_{\text {hard }}\right)
$$

where $\Gamma_{S L}$ denotes the order $\alpha_{s}$ partial decay width for (11). Because the deviations of the moduli of the parameters $\alpha$ and $\beta$ from their SM values are not expected to be large we use $\Gamma_{S L}^{S M}$ for the normalisation in (10). We use $\Gamma_{S L}^{S M}=0.168 \mathrm{GeV}$ and 0.153 $\mathrm{GeV}$ to lowest order and to order $\alpha_{s}$, respectively. We get $\langle\mathcal{O}\rangle=c \operatorname{Im}\left(\alpha^{*} \beta\right) S$, where $c=0.0050$ at tree level and $c=0.0042$ including the order $\alpha_{s}$ corrections. This implies that the effect is too small in order to obtain an interesting sensitivity to $\operatorname{Im}\left(\alpha^{*} \beta\right)$ (i.e. at the percent level) from semileptonic top quark decays.

In summary we have computed, for a $t b W$ vertex with left- and right-handed components, double differential lepton distributions to order $\alpha_{s}$ for polarised semileptonic top decay, and we presented the QCD corrections in terms of compact parameterisations. These formulae should be a useful module in the theoretical description of $t$ and/or $\bar{t}$ production and decay at next-to-leading order in $\alpha_{s}$, including nonstandard top couplings.

\section{Acknowledgments}

We would like to thank A. Brandenburg for useful comments.

\section{References}

[1] T. Affolder et al. (CDF collab.), Phys. Rev. Lett. 84 (2000) 216;

P. Merkel, talk given at the International Europhysics Conference on High Energy Physics, Aachen, July 2003.

[2] M. Beneke et al., arXiv:hep-ph/0003033, in: CERN Yellow Report, CERN 2000-004, G. Altarelli, M. Mangano (Eds.) 
[3] J. A. Aguilar-Saavedra et al. [ECFA/DESY LC Physics Working Group Collaboration], arXiv:hep-ph/0106315.

[4] P. L. Cho and M. Misiak, Phys. Rev. D 49 (1994) 5894 arXiv:hep-ph/9310332.

[5] K. Fujikawa and A. Yamada, Phys. Rev. D 49 (1994) 5890.

[6] M. Hosch, K. Whisnant and B. L. Young, Phys. Rev. D 55 (1997) 3137 arXiv:hep-ph/9607413.

[7] F. Larios, M. A. Perez and C. P. Yuan, Phys. Lett. B 457 (1999) 334 arXiv:hep-ph/9903394.

[8] D. Espriu and J. Manzano, Phys. Rev. D 65 (2002) 073005 arXiv:hep-ph/0107112; $\quad$ Phys. Rev. $\quad$ D $\quad 66 \quad$ (2002) 114009 arXiv:hep-ph/0209030.

[9] F. del Aguila and J. A. Aguilar-Saavedra, Phys. Rev. D 67 (2003) 014009 arXiv:hep-ph/0208171.

[10] M. Schmitt, in: DESY Orange Report DESY 96-123D, P. Zerwas (Ed.)

[11] E. Boos, M. Dubinin, M. Sachwitz and H. J. Schreiber, Eur. Phys. J. C 16 (2000) 269 arXiv:hep-ph/0001048.

[12] J. P. Ma and A. Brandenburg, Z. Phys. C 56 (1992) 97.

[13] M. Jezabek and J. H. Kühn, Phys. Lett. B 329 (1994) 317 arXiv:hep-ph/9403366.

[14] C. A. Nelson, B. T. Kress, M. Lopes and T. P. McCauley, Phys. Rev. D 57 (1998) 5923 arXiv:hep-ph/9706469.

[15] B. Grzadkowski and Z. Hioki, Nucl. Phys. B 585 (2000) 3 arXiv:hep-ph/0004223.

[16] A. Czarnecki, M. Jezabek and J. H. Kühn, Nucl. Phys. B 351 (1991) 70; M. Jezabek and J. H. Kühn, Nucl. Phys. B 320 (1989) 20.

[17] A. Czarnecki and M. Jezabek, Nucl. Phys. B 427 (1994) 3 arXiv:hep-ph/9402326. 


\section{FIGURE CAPTIONS}

Fig. 1. The order $\alpha_{s}$ contributions to the polarisation independent part of the lepton distributions (3), (44).

Fig. 2. The order $\alpha_{s}$ contributions to the polarisation dependent part of the lepton distributions (31), (44). 


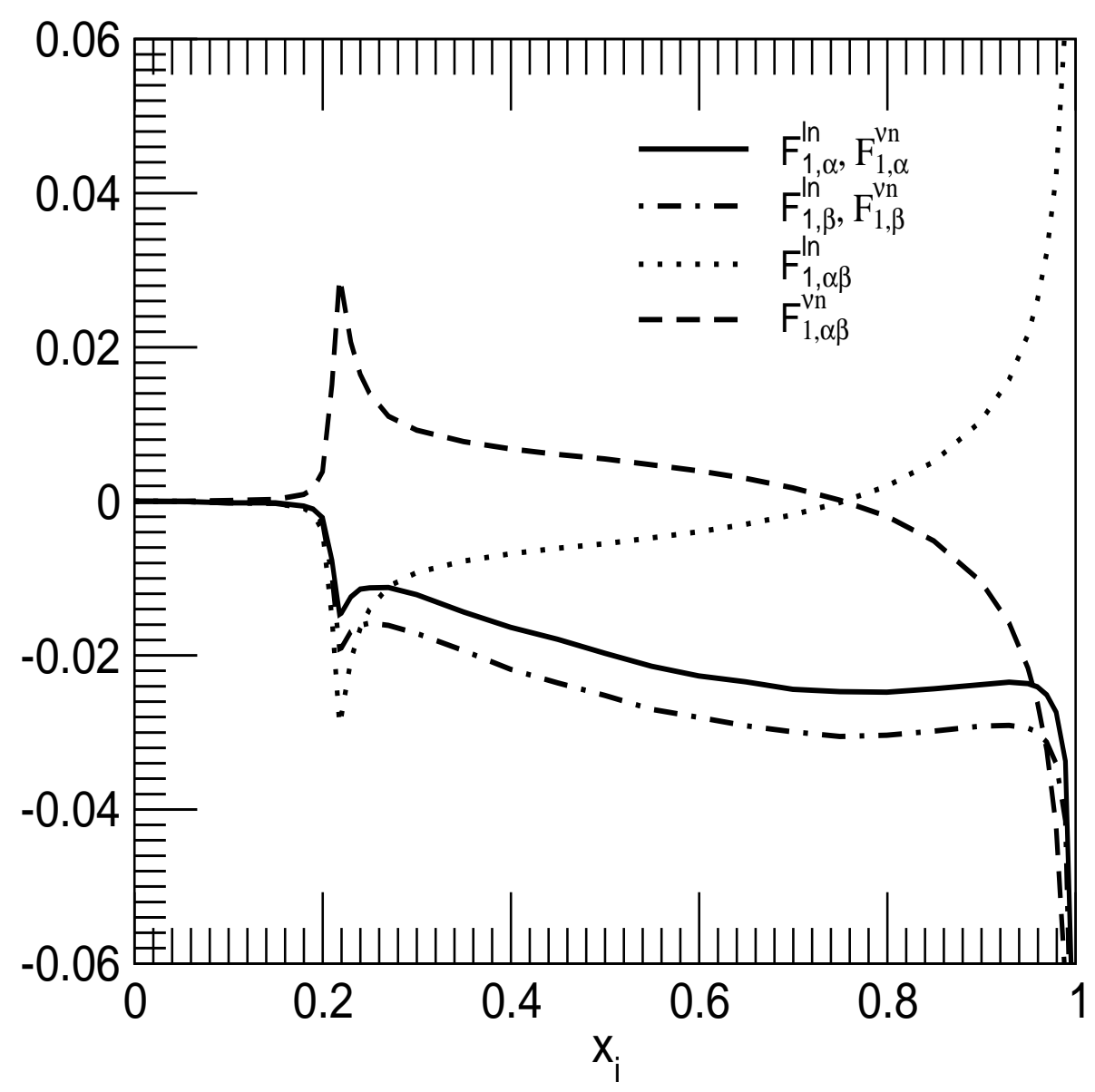

Figure 1: 


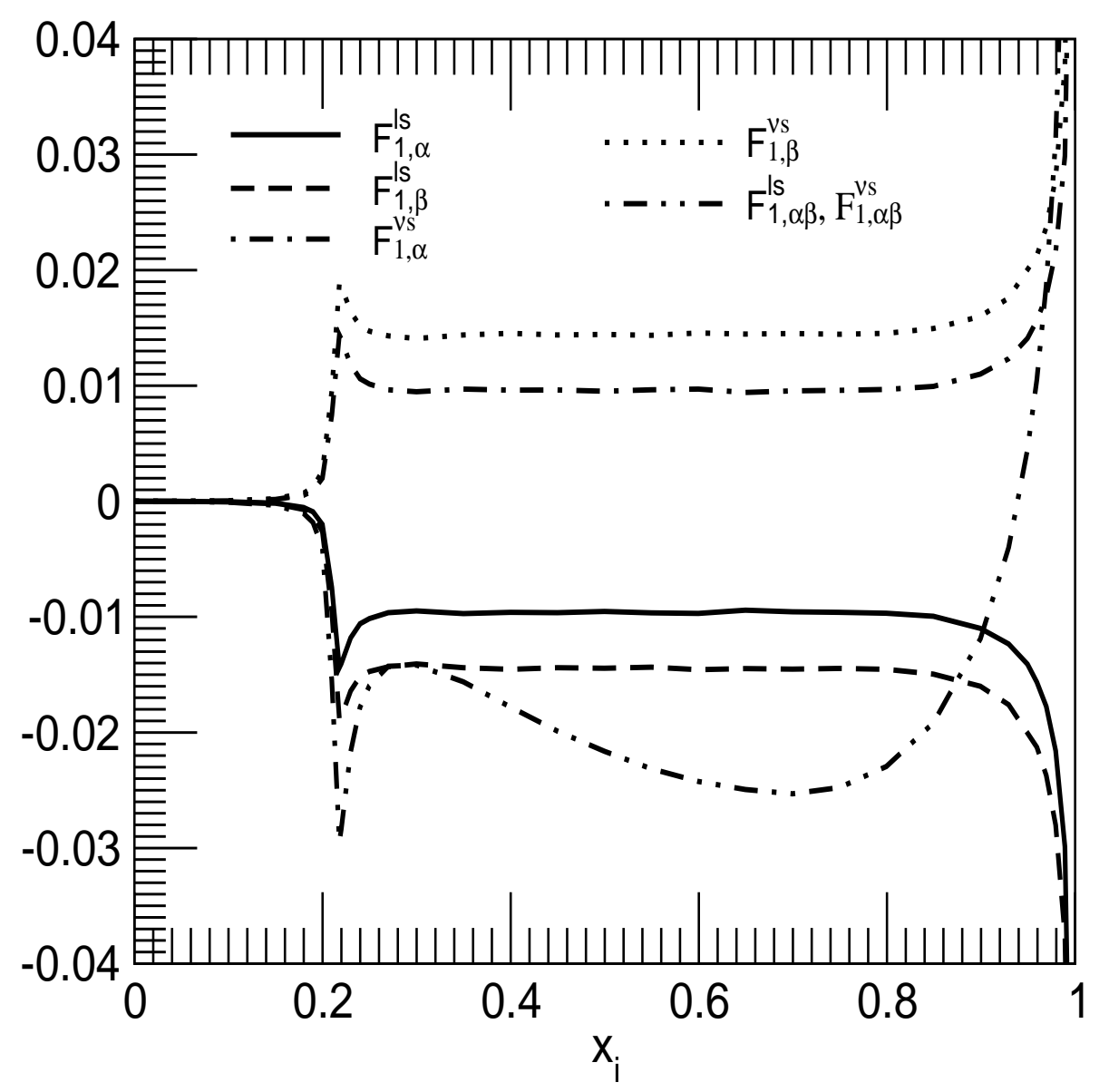

Figure 2: 